\title{
Analysis of Polka Contention Manager for use in Multicore Hard Real-Time Systems
}

\author{
Adrien Quillet \\ adrien.quillet@ls2n.fr \\ LS2N, UMR CNRS 6004 \\ Ecole Centrale de Nantes \\ Nantes, France
}

\author{
Audrey Queudet \\ audrey.queudet@ls2n.fr \\ LS2N, UMR CNRS 6004 \\ University of Nantes \\ Nantes, France
}

\author{
Didier Lime \\ didier.lime@ls2n.fr \\ LS2N, UMR CNRS 6004 \\ Ecole Centrale de Nantes \\ Nantes, France
}

\begin{abstract}
Transactional memory (TM) draws the attention of both academic and development groups; indeed this concept offers an alternative to lock-based approaches, easing programmers' work. Despite the large amount of investigations around this topic, the question of the correctness of most TM implementations remains open. More specifically, the lack of upper bounds on the execution time of transactions prevents the use of TM in real-time systems. To address this issue, we introduce new realistic assumptions relative to real-time systems, which allow to ensure wait-freedom guarantees progress (i.e. all transactions progress) when Polka contention manager is considered. In that context, through a thorough formalization of the system, we prove upper bounds both on the number of abortions and on the execution time of transactions.
\end{abstract}

\section{CCS CONCEPTS}

- Computer systems organization $\rightarrow$ Real-time operating systems; Multicore architectures.

\section{KEYWORDS}

real-time systems, non-blocking synchronisation, software transactional memory

\section{ACM Reference Format:}

Adrien Quillet, Audrey Queudet, and Didier Lime. 2020. Analysis of Polka Contention Manager for use in Multicore Hard Real-Time Systems. In 28th International Conference on Real-Time Networks and Systems (RTNS 2020), June 9-10, 2020, Paris, France. ACM, New York, NY, USA, 11 pages. https://doi.org/10.1145/3394810. 3394825

\section{INTRODUCTION AND MOTIVATION}

Real-time systems are increasingly used in electronic devices to control physical events. Those systems are designed to satisfy three objectives: (i) logical determinism, so runs with

Permission to make digital or hard copies of all or part of this work for personal or classroom use is granted without fee provided that copies are not made or distributed for profit or commercial advantage and that copies bear this notice and the full citation on the first page. Copyrights for components of this work owned by others than ACM must be honored. Abstracting with credit is permitted. To copy otherwise, or republish, to post on servers or to redistribute to lists, requires prior specific permission and/or a fee. Request permissions from permissions@acm.org.

RTNS 2020, June 9-10, 2020, Paris, France

() 2020 Association for Computing Machinery.

ACM ISBN 978-1-4503-7593-1/20/06 . . \$15.00

https://doi.org/10.1145/3394810.3394825 the same inputs always produce the same results, (ii) temporal determinism which guarantees that temporal constraints of every system action will be satisfied, and (iii) reliability which ensures the system is always available.

In the environment in which real-time systems operate, events usually take place concurrently. In order to handle them properly (i.e. to guarantee that all timing constraints will be satisfied), they resort to concurrent programming: each monitored event is managed by a processing entity called task. As multiple tasks can run in parallel over different processor cores, the underlying platform on which the system executes must provide both synchronization and communication mechanisms. In particular, an important aspect is the guarantee of data consistency. Resources shared between several tasks typically have a limit on the number of simultaneous accesses; as a consequence it is possible that a task can not progress due to an unavailable resource. The system must then efficiently manage resources to avoid both deadlocks and starvation while ensuring all the tasks still meet their deadlines.

One common way to protect concurrent modifications to shared memory is to use locks which are well known to be subject to priority inversion and deadlocks. Considering multicore platforms, another issue inherent to the use of such blocking mechanisms is that parallelism can be severely impacted since concurrent tasks competing for the same lock can be blocked, thus reintroducing some sequential execution in the parallel application. Recently [21], spin locks were studied for parallel real-time tasks in where each parallel task is scheduled exclusively on several pre-assigned processors (i.e., by the federated scheduling approach). This reduces the need for locks or scheduling to synchronize concurrent access (through shared resources may introduce locking as considered by Dinh et al. [5]). The authors developed new schedulability analysis techniques for parallel tasks with spin locks, and analysed blocking times but the analysis is pessimistic.

Another way to manage shared resources is to use the concept of transactional memory [16], which aims to significantly ease development and maintenance of concurrent programs. Transactional memory implementations exist both in hardware (HTM - Hardware Transactional Memory), in software (STM - Software Transactional Memory), or under schemes that combine both hardware and software (Hybrid TM - Hybrid Transactional Memory). In HTM systems, transactional memory support is implemented by modifying the data-cache protocols to support version management and conflict detection. The close synergy of the hardware with the processor 
core and cache allow these systems to provide very high levels of performance. Nevertheless, this type of TM system is usually bound by some sort of capacity contraints, e.g. the hardware can handle a specific fixed read-/write-set size. In contrast, STMs allow to build very flexible prototypes, as the full concurrency control is implemented in software. In return, STMs have a reputation for being slower than HTMs. However, considering the conceptual benefits of STMs, we will focus only on STMs in this paper. They allow programmers to embed a sequence of operations applied to shared resources into a transaction. Every transaction accesses shared data in memory without interfering with other transactions, and appears to them as executed atomically. To deal with inconsistencies, transactional memories usually use contention managers $(\mathrm{CM})$ to resolve conflicts between transactions. Contention managers allow some transactions to commit (i.e. the data modifications are then made permanent), and force other conflicting ones to abort (i.e. all the modifications are discarded and the transaction restarts from the beginning), based on their internal policy.

Because software transactional memories hide to developers the way atomicity is obtained, it is essential to prove the correctness of their mechanisms, especially when used in realtime systems. In this paper, we introduce new assumptions on the execution time of transactions. We formally prove that the use of the lock-free contention manager Polka [24] in such constrained systems is sufficient to guarantee that every transaction progresses, bringing in upper bounds on the execution time and the number of abortions of a transaction.

The rest of this paper is organized as follows. We first present related works about transactional memories in Section 2. We then recall the main concepts of STM in Section 3. Section 4 introduces the formalization of the system we study. Section 5 focuses on Polka contention manager and contains formal proofs about its progress property. Finally, Section 6 concludes the paper.

\section{RELATED WORK}

\subsection{Transactional Memory}

Herlihy et al. were the first to propose a hardware based transactional synchronization methodology [16] that uses the cache coherence protocol to detect conflicts. Since then, the research community has produced many different HTMs $[4,13,14,19]$ that are more efficient. Chip vendors have increasingly begun to integrate HTM in their processors like the AMD's Advanced Synchronization Facility (ASF) [17]. Transactional synchronization extensions (TSX) whose performance was recently analysed in [22] were also adopted by Intel for its processors in the year 2012. However, since HTMs require hardware facilities, they are not suitable for most of multicore processors. Other software-based implementations using common atomic instructions such as CAS (Compareand-Swap) or LL/SC (Load Linked/Store Conditional) [20], were first proposed by Shavit and Touitou in [26]. By nature, they are less efficient than HTM but they can be used with all multicore processors.

\subsection{TM Correctness and Timing Analysis}

The first steps to formally verify TM systems were made through Greedy [10] and FTGreedy [9] CM proposals. Guerraoui et al. proposed a new and robust $\mathrm{CM}$ that provides provable properties (e.g. every transaction commits within bounded time). They also introduced the notion of opacity [11], a correctness criterion for TM implementations.This idea was extended by Imbs et al. in [18]. Schoeberl et al. proposed Real-Time Transaction Memory (RTTM) [25], a new hardware-implemented synchronization paradigm for hard real-time systems. Their demonstrate that, provided the transaction conflict resolve time is bounded, tasks will meet their deadlines. Nevertheless, they do not provide any guarantees that transactions will complete successfully.

Sarni et al. presented Real-Time STM [23], a TM protocol ensuring lock-freedom, a weaker property than wait-freedom, that does not guarantee that all transactions will meet their deadlines, thus being suitable only for soft real-time systems.

Upper bounds for the number of transaction retries in a real-time system were provided by Schoeberl et al. [25] and El-Shambakey et al. $[6,7]$. However, the conditions were very simple, with periodic tasks and one transaction performed by each task.

Cotard et al. [3] proposed a new STM for hard real-time systems, that relies on a wait-free protocol that enforces progress of every task and that can be integrated in the WCET of tasks during the timing analysis of the system. However, the approach is restricted to transactions that manipulate objects in a single access mode, either read or write.

Barros et al. [1] addressed the response time analysis of hard real-time tasks, which share STM data under partitioned scheduling strategies. They assume that transactions are serialised according to their arrival time and they are managed by following a FIFO contention manager for real-time systems (FIFO-CRT) [2].

Although those works are close in purpose to ours, none of them prove the wait-freedom property (i.e. all trans- actions progress) among existing CMs.

\section{BACKGROUND MATERIAL}

\subsection{Software Transactional Memory Concepts}

A transaction, as originally formalized in [8], is based on the notion of synchronization point, which represents a durable and consistent state of a computer system. This notion relies on the four ACID properties [12]:

- Atomicity. The sequence of operations either all occur (the transaction commits), or nothing occur (the transaction aborts), which makes the sequence of operations indivisible.

- Consistency. The transaction starts and ends in a valid system state.

- Isolation. A transaction being executed is unaware of other executing transactions.

- Durability. Once a transaction has been committed, it will remain so. 
During its execution, a transaction will manipulate one or several data, either in reading or writing modes. This set of data is called the transaction dataset.

Definition 3.1. Two transactions are said in contention if they both access a same resource during their execution and one of them may update it.

Definition 3.2. Two transactions are said in conflict if they performed an operation on the same resource and one of them updated it.

An STM needs a data version management policy in order to maintain old values, needed to abort the transaction, and new values, needed to commit the transaction. Concurrency control is based on a conflict detection policy which defines the rules triggering the abortion of a transaction. There are two approaches: (i) with the eager approach, conflicts are detected and resolved as soon as they occur, (ii) with the lazy approach, conflicts detection and resolution occur later, generally before trying to commit the transaction. Finally, a transactional memory typically has a contention manager module [15], which is in charge of resolving conflicts between transactions. Depending on its contention policy (see [24] for a survey), the CM decides which of the conflicting transactions can continue, and as a result, which transaction(s) must abort.

\subsection{Polka Contention Manager}

The Polka contention manager [24] favors the transactions which performed the greater number of operations. The chosen criterion to quantify how much work has been done at a given time is the number of data already accessed either in reading or writing mode; it is called karma and is denoted by $k$. It is a component of the state of the transaction. Transactions start with a zero karma which is incremented by one at each first access to a data (first access is renewed each time the transaction starts or restarts its operations) or at each abortion of the transaction. When a transaction aborts, the maximum number of times it may restart is bounded by the difference in karma between itself and its enemy. Moreover, Polka introduces an exponential backoff between successive transaction restarts. Every aborted transaction waits for a random duration that increases exponentially. When a transaction commits, its karma returns to zero.

\section{MODELS AND TERMINOLOGY}

\subsection{System Model}

We consider a platform made of $m$ identical processor cores $\pi=\left\{\pi_{1}, \ldots, \pi_{m}\right\}$, so the maximum number of task instances that can run in parallel is $m$.

4.1.1 Time Representation. The system time sequence $t=$ $t_{i 1 \leq i}$ is a sequence of countable instants. We denote by $\mathbb{T}$ the domain of $t$. At any time, the difference between two subsequent instants $t_{i+1}$ and $t_{i}$ is 1 ; this minimum period is called a time unit.

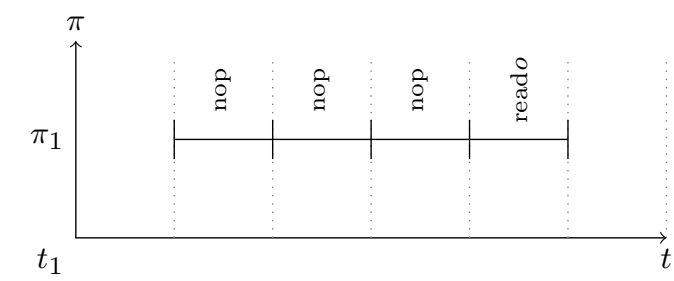

Figure 1: Execution model for a 4 time units operation

4.1.2 Objects. We assume that every shared resource managed by an STM is actually manipulated with an object. We denote by $O_{\mathcal{S}}$ the finite set of system objects such that $O_{\mathcal{S}}=O_{\bar{\Sigma}} \cup O_{\bar{\Sigma}}$, where $O_{\bar{\Sigma}}$ and $O_{\Sigma}$ denote the finite set of objects used only outside (resp. inside) an explicit transaction. The final set of all possible values for system objects is denoted by $\Upsilon^{\perp}=\Upsilon \cup\{\perp\}$ where $\Upsilon$ represents the finite set of all possible defined values for the objects whereas $\perp$ stands for the undefined value. The value of objects is given by a function Val $_{\mathcal{S}}: O_{\mathcal{S}} \rightarrow \Upsilon^{\perp}$ such that:

$$
\mathrm{Val}_{\mathcal{S}} O=x \text { iff } \mathrm{x} \text { is the value of object } \mathrm{o}
$$

4.1.3 Operations. Let $\Theta_{\mathcal{S}}$ denote the finite set of system operations. We consider three possible operations:

- reado: $\forall o \in O_{\mathcal{S}}$, it returns the value of object $o$ (i.e. $\left.\mathrm{Val}_{\mathcal{S}}\right)$;

- writeo, $x: \forall o \in O_{\mathcal{S}}, x \in \Upsilon^{\perp}$, it writes the value $x$ in object $o$;

- nop: it stands for the idempotent operation, thus leaving the system in the same state as it was before.

We assume that each operation requires one time unit to be performed. Note that every other kind of operations (e.g. arithmetic ones) can be abstracted to a sequence of read(), write() and nop() operations. In order to model an operation that takes more than one time unit to execute, the operation has to be preceded by nop() operations. As a result, to model an operation that requires $k$ time units, it must be preceded by $k-1$ idempotent operations, as illustrated in Figure 1.

\subsection{Task Model}

Let $T_{\mathcal{S}}$ denote the finite set of system tasks. A task $\tau_{i}$ is a tuple $\left\langle\pi_{i}, \phi_{i}, T_{i}, \Sigma_{i}\right\rangle$, where $\pi_{i}$ is the processor core on which all instances (or jobs) of $\tau_{i}$ will be executed, $\phi_{i}$ is the offset of first activation of the task, $T_{i}$ is the minimum period between two activations of the task and $\Sigma_{i}$ is the finite set of transactions launched during every job.

Each system task $\tau_{i}$ produces an infinite number of successive jobs $\mathrm{J}_{i, j}$, with $j \in \mathbb{N}^{*}$, as illustrated in Figure 2 . All jobs of a given task are assumed independent. Job $\mathrm{J}_{i, j}$ corresponds to the $j^{\text {th }}$ activation of task $\tau_{i}$. We denote by $r_{i, j} \geq r_{i, j-1}+T_{i}$ the release time of job $\mathrm{J}_{i, j}$. The release time $r_{i, 0}$ of the first job of task $\tau_{i}$ is equal to $\phi_{i}$.

Definition 4.1. A job is said active as soon as released. 


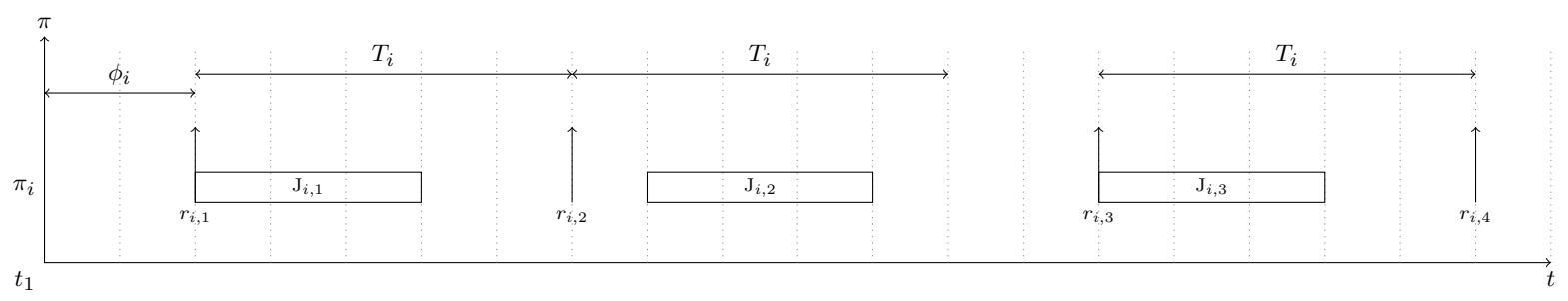

Figure 2: Execution of a task $\tau_{i}=\left\langle\pi_{i}, \phi_{i}, T_{i}, \Sigma_{i}\right\rangle$

At any time, there exists at most one active job for a given task. An active job can be preempted at some time $t_{n}$, except if it is executing a transaction.

\subsection{Transaction Model}

First, we assume that a transaction only belongs to one task.

4.3.1 Formalization. A transaction $\sigma_{i}$ is a tuple $\left\langle b_{i}, N_{i}, \Omega_{i}, O_{i}\right\rangle$, where $b_{i}$ represents its start time, $N_{i}$ is the number of operations in the transaction, $\Omega_{i}$ is its dataset with $\Omega_{i} \subseteq O_{\Sigma}$ and $O_{i}$ is the transaction private memory, which contains copies of the objects used by the transaction. This private memory is only visible to the transaction itself. Hence, a transaction works on its own copies of the objects and if it successfully commits, then the commit phase is completed by copying the private modifications into the shared memory.

We denote by $\eta_{i}=\left\langle\xi_{i}, n_{i}, \omega_{i}, c_{i}, \mathrm{Val}_{i}\right\rangle$ the state of $\sigma_{i}$, where $\xi_{i}$ is the current status of $\sigma_{i}, n_{i}$ is the actual number of performed operations, $\omega_{i}$ is its finite set of already opened objects with $\omega_{i} \subseteq \Omega_{i}, c_{i}$ is its internal clock starting at 0 and increased by one at each performed operation, and $\mathrm{Val}_{i}$ from $O_{i} \rightarrow \Upsilon^{\perp}$, is the function giving the value of an object from its transaction private memory. There exists 5 different transaction status:

- NotStarted is the status of a transaction that has never started;

- Running is the status of a processing transaction which has at least one more operation to perform;

- Checking is the status of a transaction which performed all its operations, and is verifying that its modifications will not leave the system in an inconsistent state;

- Aborted is the status of a transaction which has been aborted;

- Committed is the status of a transaction that has completed its execution. All committed modifications are visible to the entire system.

Definition 4.2. A transaction is said processing if its status is either Running, Checking or Aborted.

Definition 4.3. A transaction is said active if its status is either Running or Checking.

We denote by $\operatorname{Exec}_{\Sigma} t_{n}$ the set of processing transactions at time $t_{n}$, and by $\operatorname{Active}_{\Sigma} t_{n}$ the set of active transaction the same time.

We denote by $\Sigma$ the finite set of system transactions and by $\mathcal{N}=\left\{\eta_{1}, \ldots, \eta_{|\Sigma|}\right\}$ the finite set of transaction states.
Let $\Theta_{\Sigma}$ denote the finite set of operations specific to transactions, such that it exists 5 different operations related to transactions:

- start: starts transaction $\sigma_{i}$, initializing all its parameters properly.

- restart: restarts transaction $\sigma_{i}$ after an abortion. This operation resets all transaction parameters except its start time which remains unchanged.

- check: brings transaction $\sigma_{i}$ into checking phase. This operation checks if all previously opened objects keep the same values, thus detecting potential conflicts with other transactions.

- abort: cancels all modifications made inside the transaction itself.

- commit: makes all modifications made by the transaction visible for the entire system.

Note that depending on other currently executed transactions and on the read-/write-set sizes, the execution time of check, abort, and commit operations may vary.

Transactions can also perform any operation of $\Theta_{\mathcal{S}}$. Accordingly, the domain of arguments of system operations is extended to $O_{\mathcal{S}} \cup O_{1} \cup \ldots O_{|\Sigma|}$. The set of operations that is possible to perform from a given status is illustrated in Figure 3.

4.3.2 Properties. The system state resulting of the parallel execution of transactions is consistent only if this execution is serializable.

Definition 4.4. A parallel execution of $n$ transactions is serializable if it is equivalent to a serial execution of these $n$ transactions.

We propose to distinguish the following notion of transaction correctness.

Let TT (Turnaround Time) denote the upper bound on the time during which transactions can remain active in the system. This bound is set a priori by programmers. For instance, considering a real-time application, this upper bound may be set according to the real-time constraints of the tasks, using a worst case execution time (WCET) analysis.

Definition 4.5. A transaction is said timely correct if the maximum period between its (start()|restart()) and (abort()| commit()) operations is less than or equal to TT. Otherwise (i.e. this period is exceeded), the transaction is said timely incorrect. 
Since a transaction has to perform at least one system operation other than nop() and three specific operations (start(), check() and commit()) to be valid, the minimum value of $\mathrm{TT}$ is 4 time units.

Let $\sigma_{i} \dagger \sigma_{j}$ (resp. $\sigma_{i} \ddagger \sigma_{j}$ ) denote the fact that transactions $\sigma_{i}$ and $\sigma_{j}$ are in contention (resp. in conflict). By extension, we denote by $\sigma_{i} \dagger$ Enemies $_{i}$ (resp. $\sigma_{i} \ddagger$ Enemies $_{i}$ ) the fact that $\sigma_{i}$ is in contention (resp. in conflict) with every transaction of the transaction set Enemies $i$.

Definition 4.6. The sequence of operations performed between (start()|restart()) and (abort()|commit()) operations (included) of a transaction is called cycle of execution of this transaction.

Definition 4.7. A transaction $\sigma_{i}$ progresses if, at a given time $t_{n}, \sigma_{i} \in \operatorname{Active}_{\Sigma} t_{n}$ and there exists $t_{m}>t_{n}$ such as at time $t_{m}$, the status of $\sigma_{i}$ is Committed.

For ease of reference, notations previously presented are summarized in Table 1.

\section{SCOPING POLKA CM}

In this section, we discuss how the karma values assigned by Polka CM to transactions may vary along time. In particular, we study the extreme cases for which transactions exhibit minimal or maximal karma values.

Let us first recall how conflicts are resolved in Polka CM. So, let us assume that at a given time there exists both a checking transaction $\sigma_{i}$ and a set Enemies Ef transactions conflicting with $\sigma_{i}$. The following two cases are therefore possible:

- $\forall \sigma_{e} \in$ Enemies $_{i}: k_{i}>k_{e}$ : every enemy transaction aborts and $\sigma_{i}$ commits,

- $\exists \sigma_{e} \in$ Enemies $_{i}: k_{i} \leq k_{e}: \sigma_{i}$ sleeps for $\Delta_{w}$ time units. Meanwhile, if a transaction from Enemies $_{i}$ commits then $\sigma_{i}$ aborts, and if every transaction from Enemies $i$ aborts, then $\sigma_{i}$ commits. At the end of its sleeping period, if $\sigma_{e}$ is still active, then $\sigma_{i}$ sleeps again only if its maximum number of sleeping periods $w_{i, e}^{\max }=$

\begin{tabular}{|c|c|}
\hline Symbol & Description \\
\hline$\pi$ & Core set of the system \\
\hline$T_{\mathcal{S}}$ & Task set of the system \\
\hline$\Sigma$ & Transaction set of the system \\
\hline$O_{\mathcal{S}}$ & Object set of the system \\
\hline$O_{\Sigma}$ & STM-managed object set \\
\hline$O_{\bar{\Sigma}}$ & Non-STM-managed object set \\
\hline$\pi_{m}$ & Core $m$ of the core set $\pi$ \\
\hline$\tau_{i}$ & Task $i$ of the task set $T_{\mathcal{S}}$ \\
\hline $\mathrm{J}_{i, j}$ & $\mathrm{~J}^{\text {th }}$ job of task $i$ \\
\hline$\sigma_{k}$ & Transaction $k$ of the transaction set $\Sigma$ \\
\hline$O_{k}$ & Private memory of transaction $k$ \\
\hline$\Omega_{k}$ & Dataset of transaction $k$ \\
\hline$b_{k}$ & Start date of transaction $k$ \\
\hline$\xi_{k}$ & Status of transaction $k$ \\
\hline$k_{k}$ & Karma of transaction $k$ \\
\hline$t_{n}$ & Time instant $n$ \\
\hline $\operatorname{Active}_{\Sigma} t_{n}$ & Set of active transactions at time $t_{n}$ \\
\hline $\operatorname{Exec}_{\Sigma} t_{n}$ & $\begin{array}{l}\text { Set of processing transactions at time } \\
t_{n}\end{array}$ \\
\hline $\mathrm{TT}$ & Turnaround Time \\
\hline
\end{tabular}

Table 1: Notations used in this paper

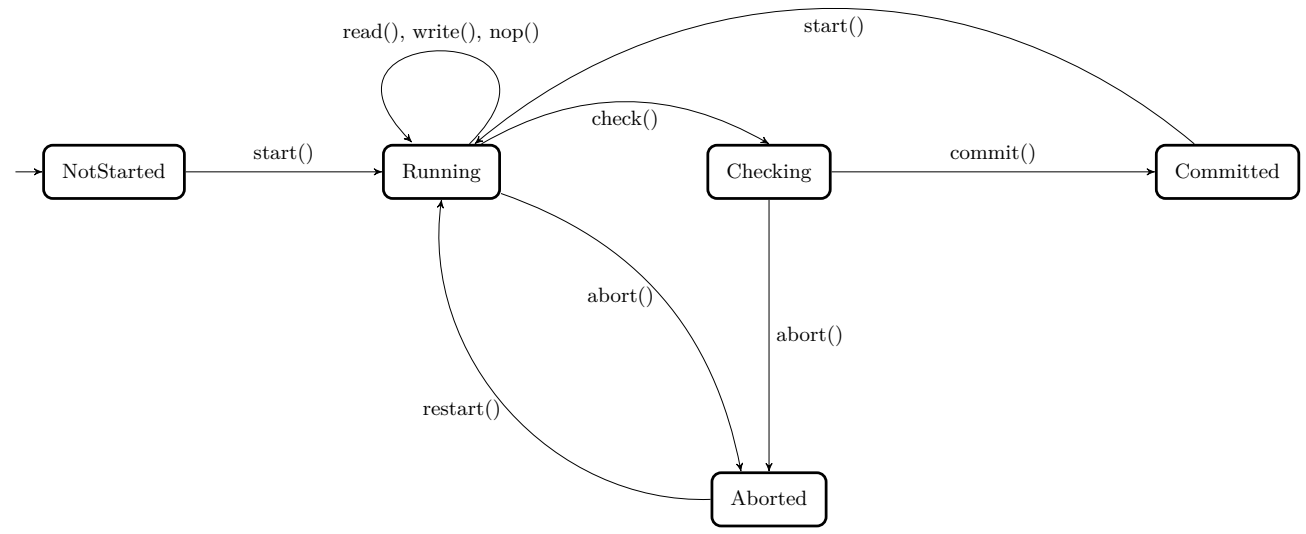

Figure 3: State-transition diagram of possible transaction status 


\subsection{Illustrative Example}

Consider a set of $(n+1)$ transactions, with $n$ slow readers and one fast writer. The execution scenario is illustrated in Figure 4. Transaction $\sigma_{w}$ writes into object $o$ and $n$ transactions read the value of this object.

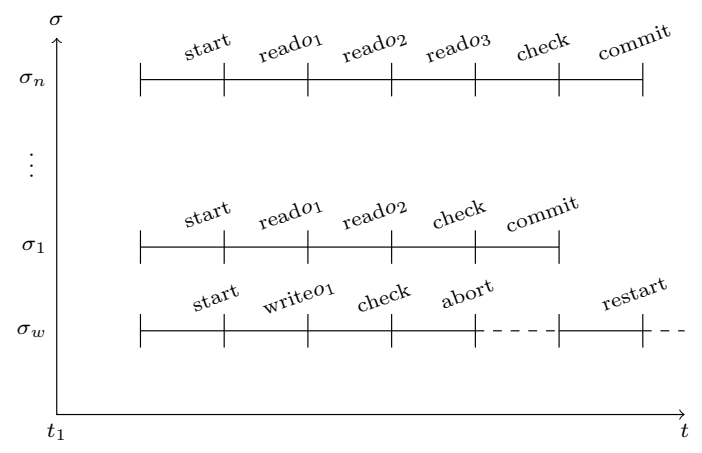

Figure 4: Fast single-writer/Slow multiple-reader scenario

Considering lazy conflict detection (check() operation occurs right before commit() operation), we can deduce the two following commit rules:

- If the writer commits, then all readers whose read() operation is performed abort;

- If a reader commits, then all readers commit and the writer aborts if its write() operation is already performed.

Let's investigate Polka arbitration among competing transactions. All transactions earn one karma unit for their execution, and one more karma unit if they abort. At the beginning of the execution, all karmas are equal to zero; the writer will commit first because it runs faster than readers. Its karma will then return to zero while the karma of all readers will be of 2 (one for the performed operation, and one for the abortion). The writer is a priori favored since it reaches validation before any reader, but Polka contention manager favors transactions that have opened the greater number of objects, their karma being incremented by one at each first object opening. As a result, if the writer's karma is lower than the karma of readers, the writer will sleep, waiting for readers to commit (see Figure 4). The only chance for readers to validate is if the writer total sleeping period is strictly greater than the difference of karma between readers and writer. If so, readers commit successfully while the writer still sleeps; the writer is aborted, its karma is increased by one and the karma of all readers return to zero.

The sleeping period being randomly drawn from an interval growing exponentially, the more readers are aborted, the more likely they are to validate, because the writer sleeping period has a chance to exponentially grow.

Without studying more specifically Polka policy, we cannot say if readers will eventually validate. They have more and more chances to commit, but if the total sleeping period of the writer indefinitely remains lower than the difference of karma between the readers and the writer, then the writer will indefinitely abort all readers. Hereafter, we focus on the worst (i.e. slowest) and best (i.e. fastest) karma accumulation for transactions in terms of speed.

\subsection{Slowest Karma Accumulation}

LEMMA 5.1. In a system using a software transactional memory whose contention manager is Polka, the slowest karma accumulation at time $t_{n}$ of a timely correct transaction $\sigma_{l}$ is given by:

$$
k_{l}=\left\lfloor\frac{t_{n}-b_{l}}{T T}\right\rfloor \times 2+\epsilon_{l} \text { with } \epsilon_{l} \in\{0,1\}
$$

Proof. Let $\sigma_{l}$ denote the timely correct transaction whose karma increases the slowest. According to Polka policy, the slowest way to increase karma is to manipulate the fewest possible objects, while taking the maximum amount of time to do it. Since we study karma accumulation, it is assumed that this transaction indefinitely aborts. Hence $\sigma_{l}$ must have a single operation to perform (so its dataset contains only one object). The timing diagram for the execution of $\sigma_{l}$ is illustrated in Figure 5.

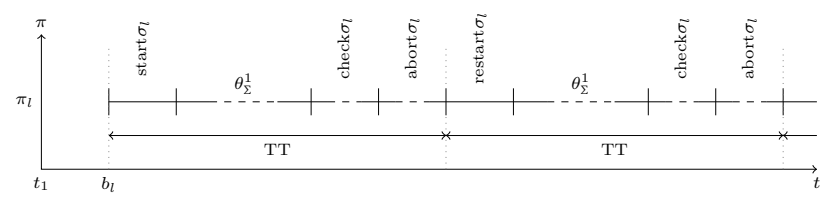

Figure 5: Slowest karma accumulation by a transaction $\sigma_{l}$

Transaction $\sigma_{l}$ increases its karma by one not only when it accesses its single object but also at each abort() operation. As a result, its karma is increased by 2 every TT time units. So, at given time, the karma of $\sigma_{l}$ is double the number of execution cycles of the transaction, in addition to the karma $\epsilon_{l}$ accumulated during the current execution cycle.

\subsection{Fastest Karma Accumulation}

LEMMA 5.2. In a system using a software transactional memory whose contention manager is Polka, whose conflict detection is lazy and in which all transactions are timely correct, the fastest karma accumulation at time $t_{n}$ of transaction $\sigma_{w}$ is given by:

$$
\begin{gathered}
k_{w}=\left\lfloor\frac{t_{n}-b_{w}}{T T}\right\rfloor \times(T T-2)+\epsilon_{w} \\
\text { with } \epsilon_{w} \in\{0, \ldots, n\}
\end{gathered}
$$

Proof. Let $\sigma_{w}$ denote the timely correct transaction whose karma increases the fastest. According to Polka policy, the fastest way to accumulate karma is to access as many various objects as possible in a minimum amount of time. Because (start()/restart()) and check() operations do not increase karma, a timely correct transaction accessing as many objects as possible in TT time units eventually has a greater karma than a transaction accessing a single object in one time unit that is aborted and restarted over a period of TT time 


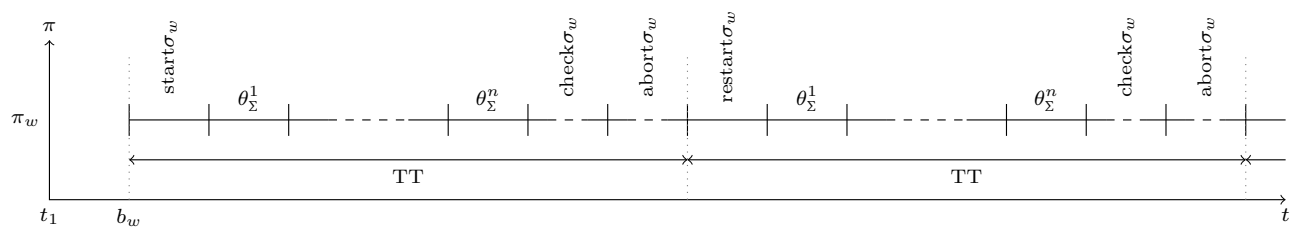

Figure 6: Fastest karma accumulation by a transaction $\sigma_{w}$

units. As previously, it is also assumed that this transaction indefinitely aborts.

Transaction $\sigma_{w}$ thus accesses to a maximum number of objects over its interval of correctness, keeping the remaining time units to perform operations (start()/restart()), check() and $($ abort ()$)$. The timing diagram for the execution of $\sigma_{w}$ is illustrated in Figure 6.

Transaction $\sigma_{w}$ increases its karma by one for each performed operation or abort() operation. Consequently, its karma is increased by $(\mathrm{TT}-2)$ every TT time units. So, at a given time, the karma of $\sigma_{w}$ is $(\mathrm{TT}-2)$ times the number of execution cycles of the transaction, in addition to the karma $\epsilon_{w}$ accumulated during the current execution cycle.

\subsection{Achieving the Greatest Karma}

LEMMA 5.3. In a system using a software transactional memory whose contention manager is Polka, whose conflict detection is lazy and in which all transactions are timely correct, the maximum possible karma value is

$$
\begin{gathered}
k_{\mathcal{S}}^{\max }=(\min (|\pi|,|\Sigma|)-1) \times(T T-2)+\epsilon \\
\text { with } \epsilon \in\{0, \ldots, n\}
\end{gathered}
$$

Proof. Let us argue by contradiction, assuming that there is no upper bound on the karma.

First, let us study the trivial case where there is only one transaction in the system. A single transaction cannot be in conflict with itself and thus cannot be aborted. As a consequence, this single transaction always commits and always achieves the greatest karma which is the number of objects it accessed. Hence there exists an upper bound $\epsilon$ on the karma equals to the number of objects opened by the transaction during its execution.

Let now consider that there are at least two timely correct transactions $\sigma_{w}$ and $\sigma_{w^{\prime}}$ in the system, such that there is no upper bound on their karma. Assuming that $k_{i} t_{z}$ denote the karma of a transaction $\sigma_{i}$ at time $t_{z}$, this can be expressed by the following property:

$$
\begin{aligned}
& \forall t_{n}, \exists t_{m}>t_{n}: \\
& \qquad\left(k_{w} t_{n}<k_{w} t_{m}\right) \vee\left(k_{w^{\prime}} t_{n}<k_{w^{\prime}} t_{m}\right)
\end{aligned}
$$

Let us prove this property is false.

The lack of upper bound on the karma means that there is no upper bound on the number of abortions of a transaction. As a consequence, there always exists a transaction which karma is greater than the previous greatest karma value. Note that the transaction with the greatest karma can vary over time. To examine the evolution of karma, we consider the worst-case execution for system transactions. We assume that all transactions accumulate karma the fastest way and share the same dataset; the timing diagram of their execution is illustrated in Figure 6. To maximize contention between transactions, we also consider the execution case illustrated in Figure 7. The maximum number of concurrent transactions is the minimum between the number of system transactions and processor cores, and all transactions start at the same time.

Let $\sigma_{w}$ denote the transaction executed on processor core $\pi_{w}$. Transaction $\sigma_{w}$ reaches its checking phase before any

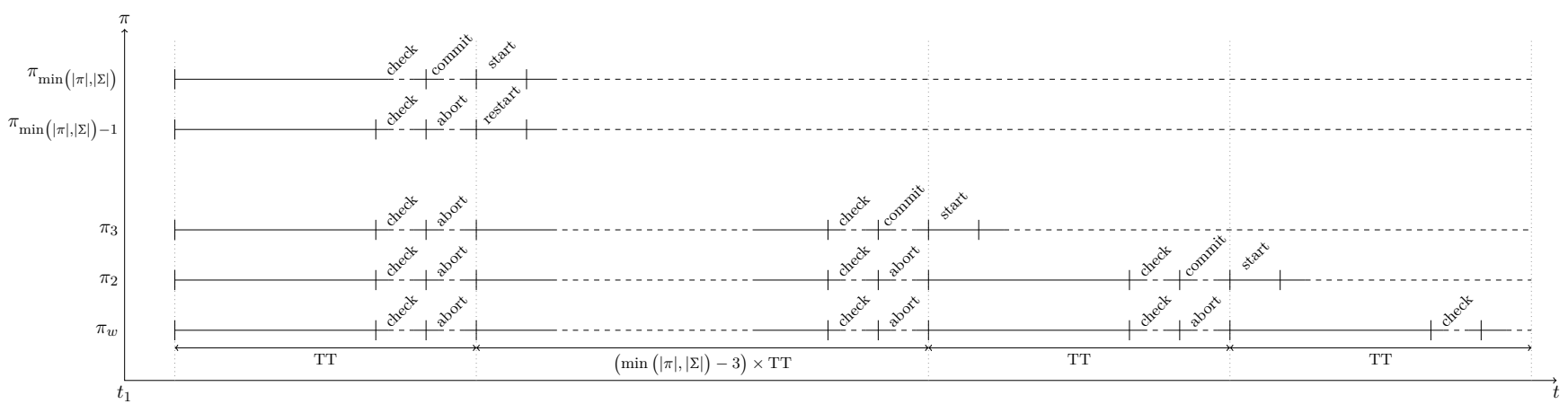

Figure 7: Worst-case contention case for a fast accumulation karma transaction $\sigma_{w}$

All transactions start at the same time and are based on the same model (as illustrated in Figure 6). As a result, if a transaction commits, it forces the abortion of all other transactions. 
other transaction; all transactions having the same karma, $\sigma_{w}$ has to sleep, waiting for other transactions to commit or abort. According to the execution case, all transactions are in conflict, so only one transaction will be allowed to commit. The last transaction reaching its checking phase will commit, and other transactions will abort, particularly $\sigma_{w}$. After a bounded number of abortions and always according to the execution pattern illustrated in Figure $7, \sigma_{w}$ will possess the greatest karma in the system. Let $\sigma_{w^{\prime}}$ denote the transaction that possesses the second greatest karma in the system; it is the transaction that committed first in the system. According to Lemma 5.2 , at time $t_{n}$, the karma of $\sigma_{w^{\prime}}$ can be expressed as:

$$
\begin{gathered}
k_{w^{\prime}}=\left\lfloor\frac{t_{n}-b_{w^{\prime}}}{\mathrm{TT}}\right\rfloor \times(\mathrm{TT}-2)+\epsilon_{w^{\prime}} \\
\text { with } \epsilon_{w^{\prime}} \in\{0, \ldots, n\}
\end{gathered}
$$

while the karma of $\sigma_{w}$ is:

$$
\begin{gathered}
k_{w}=\left\lfloor\frac{t_{n}-b_{w}}{\mathrm{TT}}\right\rfloor \times(\mathrm{TT}-2)+\epsilon_{w} \\
\text { with } \epsilon_{w} \in\{0, \ldots, n\}
\end{gathered}
$$

Since $\sigma_{w}$ has executed one more execution cycle than $\sigma_{w^{\prime}}$ and considering they have the same execution pattern, the karma of $\sigma_{w}$ can also be expressed as:

$$
\begin{aligned}
k_{w} & =\left\lfloor\frac{t_{n}-b_{w^{\prime}}-\mathrm{TT}}{\mathrm{TT}}\right\rfloor \times(\mathrm{TT}-2)+\epsilon_{w^{\prime}} \\
& =\left(\left\lfloor\frac{t_{n}-b_{w}}{\mathrm{TT}}\right\rfloor+1\right) \times(\mathrm{TT}-2)+\epsilon_{w}
\end{aligned}
$$

As a result, the maximal difference of karma between $\sigma_{w}$ and $\sigma_{w^{\prime}}$ is $(\mathrm{TT}-2)$. We then distinguish two possible cases:

- case $(i): \sigma_{w^{\prime}}$ tries to commit first.

- case (ii): $\sigma_{w}$ tries to commit first.

case (i): If $\sigma_{w^{\prime}}$ tries to commit first, then $\sigma_{w^{\prime}}$ will sleep, waiting for $\sigma_{w}$, because the karma of $\sigma_{w}$ is greater than its karma. The difference of karma between $\sigma_{w}$ and $\sigma_{w^{\prime}}$ being $(\mathrm{TT}-2), \sigma_{w^{\prime}}$ will sleep at least $(\mathrm{TT}-2)$ time units before aborting $\sigma_{w}$. Transaction $\sigma_{w}$ being in checking phase too, and considering its execution pattern, $\sigma_{w^{\prime}}$ only has one time unit to abort $\sigma_{w}$ to commit, i.e. TT $-2 \leq 1$, that is equivalent to $\mathrm{TT} \leq 3$. By definition, $\mathrm{TT} \geq 4$. As a consequence, $\sigma_{w^{\prime}}$ cannot commit before $\sigma_{w}$.

case (ii): If $\sigma_{w}$ tries to commit first, then $\sigma_{w}$ will commit because it has the greatest karma.

In both cases, the karma of $\sigma_{w}$ at $t_{n}$ allows it to commit. If $\sigma_{w}$ can commit, its karma cannot indefinitely increase. We can deduce the same fact for other transactions since they all share the same execution pattern. As a consequence, there exists an upper bound on the karma. According to the execution pattern of transactions, the greatest karma is equal to the number of concurrent transactions (not counting the current transaction) times the karma accumulated during an execution cycle (i.e. $(\mathrm{TT}-2)$ ) in addition to the karma accumulated during its last cycle (at most $n$ ). This contradicts Property 1.
LEMMA 5.4. In a system using a software transactional memory whose contention manager is Polka, whose conflict detection is lazy and in which all transactions are timely correct, the maximum period needed by a recurring aborted transaction $\sigma_{l}$ to reach the maximum possible karma $k_{\mathcal{S}}^{\max }$ is given by:

$$
\Delta_{l}^{\max }=\left(\left\lfloor\frac{k_{\mathcal{S}}^{\max }}{2}\right\rfloor+1\right) \times T T
$$

Proof. According to Lemma 5.1, the slowest karma accumulation is 2 karma units every TT time units for a timely correct transaction that always aborts. According to Lemma 5.3 , there exists an upper bound $k_{\mathcal{S}}^{\max }$ on the karma that is possible to accumulate in the system. As a consequence, there exists an upper bound on the time needed to reach this karma limit.

Assuming the worst-case execution, a timely correct transaction increases its karma by 2 every TT time units. As a consequence, after $\left\lfloor\frac{k_{\mathcal{S}}^{\max }}{2}\right\rfloor$ cycles of execution, the karma of this transaction is near or equal to $k_{\mathcal{S}}^{\max }$. Obviously, if it has not already happened, the transaction will reach the karma limit during its next cycle of execution. We obtain the upper bound established in the Lemma.

\subsection{Polka: a Wait-free CM}

THEOREM 5.5. In a system using a software transactional memory, which contention manager is Polka, and which conflict detection is lazy, if every transaction is timely correct, then every transaction progresses.

ProOF. We assume that in such a system, there exists a timely correct transaction $\sigma_{l}$ that never progresses, as expressed by the property:

$$
\exists t_{i}: \sigma_{l} \in \text { Activest }_{i} \wedge \forall t_{j}>t_{i}: \xi_{l} \neq \text { Committed }
$$

Let us prove this property is false.

Let Enemies $t_{m}$ denote the set of transactions in conflict with $\sigma_{l}$ at time $t_{m}$. We need to study four different cases:

- $\xi_{l}=$ Checking $\wedge \sigma_{l} \ddagger$ Enemies $_{l}$

(i.a) $\forall \sigma_{e} \in$ Enemies $_{l}: k_{l}>k_{e}$

(i.b) $\exists \sigma_{e} \in$ Enemies $_{l}: k_{l} \leq k_{e}$

- $\xi_{l}=$ Running $\wedge \sigma_{l} \ddagger$ Enemies $_{l}$

(ii.c) $\exists \sigma_{e} \in$ Enemies $_{l}: \xi_{e}=$ Checking $\wedge k_{e}>k_{l}$

(ii.d) $\exists \sigma_{e} \in$ Enemies $_{l}: \xi_{e}=$ Checking $\wedge k_{e} \leq k_{l}$

(i.a). Transaction $\sigma_{l}$ is in checking phase and its karma is strictly greater than the karma of every transaction conflicting with it. Transaction $\sigma_{l}$ is allowed to commit, and all conflicting transactions are aborted. It contradicts Property 2 .

(i.b). Transaction $\sigma_{l}$ is in checking phase and there is at least one transaction $\sigma_{e} \in$ Enemies $_{l}$ having a karma greater or equal to $\sigma_{l}$ 's one. Transaction $\sigma_{l}$ sleeps, waiting for $\sigma_{e}$. As a result, there are four possible cases:

(1) Transaction $\sigma_{e}$ commits. Transaction $\sigma_{l}$ aborts. Transaction $\sigma_{e}$ karma returns to zero and $\sigma_{l}$ karma is steadily increasing. According to Lemma 5.4, there exists a time 
instant $t_{n}>t_{m}$ such that $\sigma_{l}$ reaches the karma limit, i.e. $\forall \sigma_{e} \in$ Enemies $_{l} t_{n}: k_{l}>k_{e}$. We refer to cases $i . a$ and $i i . d$.

(2) Transaction $\sigma_{e}$ aborts. There are again two possible cases:

- Transaction $\sigma_{l}$ also aborts. A third transaction, in conflict with both $\sigma_{e}$ and $\sigma_{l}$, commits. As a consequence, both transactions $\sigma_{e}$ and $\sigma_{l}$ abort. According to Lemma 5.4, there exists a time instant $t_{n}>t_{m}$ such that $\sigma_{l}$ reaches the karma limit, i.e. $\forall \sigma_{e} \in$ Enemies $_{l} t_{n}: k_{l}>k_{e}$. We refer to cases $i . a$ and $i i . d$.

- Transaction $\sigma_{l}$ is still active. Transaction $\sigma_{l}$ is allowed to commit. It contradicts Property 2 .

(3) $w_{l, e}<w_{l, e}^{\max }$, i.e. the number of sleeping periods of $\sigma_{l}$, waiting for $\sigma_{e}$, is not greater than the difference between the karma of $\sigma_{l}$ and $\sigma_{e}$. Transaction $\sigma_{l}$ sleeps again, waiting for $\sigma_{e}$. We refer to cases $i . b .1, i . b .2$ and i.b.4.

(4) $w_{l, e}=w_{l, e}^{\max }$, the maximum number of sleeping periods is reached. Transaction $\sigma_{e}$ aborts and transaction $\sigma_{l}$ commits. It contradicts Property 2.

(ii.c). There is at least one checking transaction $\sigma_{e}$ in conflict with $\sigma_{l}$ having a karma strictly greater than $\sigma_{l}$ 's one. Transaction $\sigma_{l}$ aborts and $\sigma_{e}$ commits (see Figure 8). According to Lemma 5.4, there exists a time $t_{n}>t_{m}$ such that $\sigma_{l}$ reaches the karma limit, i.e. $\forall \sigma_{e} \in$ Enemies $_{l} t_{n}: k_{l}>$ $k_{e}$. We refer to cases i.a and ii.d.

(ii.d). There is at least one checking transaction $\sigma_{e}$ in conflict with $\sigma_{l}$ having a karma lower or equal to $\sigma_{l}$ 's one. $\sigma_{e}$ sleeps, waiting for $\sigma_{l}$. As a result, there are four possible cases:

(1) Transaction $\sigma_{l}$ commits. Transaction $\sigma_{e}$ aborts (see Figure 8). It contradicts Property 2.

(2) Transaction $\sigma_{l}$ aborts. Transaction $\sigma_{e}$ is allowed to commit if it is not aborted too. We refer to case ii.d.4.

(3) $w_{e, l}<w_{e, l}^{\max }$, the number of sleeping periods is not greater than the difference between $\sigma_{e}$ and $\sigma_{l}$ karmas. Transaction $\sigma_{e}$ sleeps again, waiting for $\sigma_{l}$ (see Figure 9). We refer to cases ii.d.1, ii.d.2 and ii.d.4.

(4) $w_{e, l}=w_{e, l}^{\max }$, the maximum number of sleeping periods is reached. Transaction $\sigma_{l}$ aborts and transaction $\sigma_{e}$ commits (see Figure 9).

Let investigate this last case. By hypothesis, $\sigma_{l}$ never progresses, that is in this precise situation, transaction $\sigma_{e}$ always aborts $\sigma_{l}$ by reaching the maximum number of sleeping periods. Without loss of generality, we assume the virtual worst-case execution for $\sigma_{l}$ : this transaction accumulates karma the slowest way, as described by Lemma 5.1. Transaction $\sigma_{e}$ accumulates karma the fastest way, as described by Lemma 5.2. Since both transactions are timely correct, their execution time, including sleeping periods, cannot be greater than TT. As a consequence, the maximum number of sleeping periods is TT. This is expressed by the following inequality:

$$
k_{l}-k_{e} \leq \mathrm{TT}
$$

with

$$
\begin{aligned}
& k_{l}=\left\lfloor\frac{t_{n}-b_{l}}{\mathrm{TT}}\right\rfloor \times 2+\epsilon_{l} \\
& k_{e}=\left\lfloor\frac{t_{n}-b_{e}}{\mathrm{TT}}\right\rfloor \times(\mathrm{TT}-2)+\epsilon_{e}
\end{aligned}
$$

Since $\sigma_{e}$ already aborted $\sigma_{l}$ at least one time, it started right after $\sigma_{l}$, considering the worst-case execution. So $\sigma_{e}$ karma can be expressed as:

$$
\begin{aligned}
k_{e} & =\left\lfloor\frac{t_{n}-b_{l}+1}{\mathrm{TT}}\right\rfloor \times(\mathrm{TT}-2)+\epsilon_{e} \\
& =\left\lfloor\frac{t_{n}-b_{l}}{\mathrm{TT}}\right\rfloor \times(\mathrm{TT}-2)+C_{e}
\end{aligned}
$$

with $C_{e}$ being a constant value which is equal to the sum of all constants in the previous inequality. The inequality 3 can be rewritten as:

$$
\begin{gathered}
\left(\left\lfloor\frac{t_{n}-b_{l}}{\mathrm{TT}}\right\rfloor \times 2+\epsilon_{l}\right)- \\
\left(\left\lfloor\frac{t_{n}-b_{l}}{\mathrm{TT}}\right\rfloor \times(\mathrm{TT}-2)+C_{e}\right) \leq \mathrm{TT} \\
t_{n}-b_{l}+\epsilon_{l}-C_{e} \leq \mathrm{TT} \\
t_{n} \leq \mathrm{TT}+b_{l}-\epsilon_{l}+C_{e} \\
t_{n} \leq C
\end{gathered}
$$

with $C$ being a constant value which is equal to the sum of all constants in the inequality. The system time sequence $t$ being a strictly increasing sequence without supremum, the inequality 3 is incorrect. As a consequence, there is no transaction $\sigma_{e}$ such that $\sigma_{e}$ always aborts $\sigma_{l}$. It contradicts the Property 2 .

\section{CONCLUSION AND FUTURE WORK}

While software transactional memory performances are widely studied, the question of the correctness of such mechanisms remains open. The few papers that investigated this issue actually focused on the design of new contention managers and on the modeling of transactions themselves, while there exists STM implementations with empirically efficient CMs. Motivated by this observation, we introduced new assumptions on the execution time of transactions into real-time systems in order to formally prove that, provided these systems use the existing Polka CM, they can ensure the progress of all transactions. Based on an fine-grain modeling of the whole system, we also derived upper bounds on both the execution time and the number of abortions of transactions.

Future work include the proposal of a robust variant of Polka CM, by introducing static priorities that would allow to relax the functional and timing constraints of transactions. Indeed, in the case where a transaction becomes timely incorrect, there is actually no guarantee that all timely correct transactions will progress. 


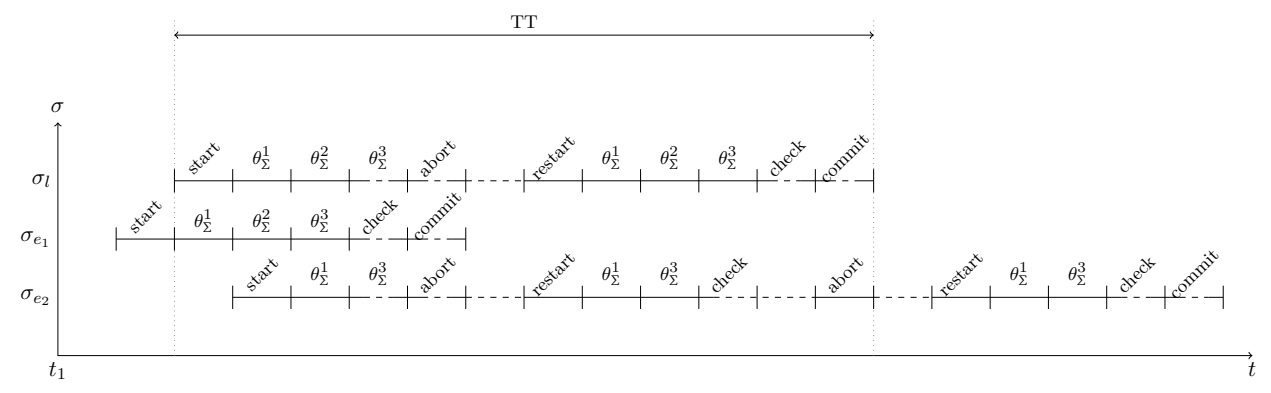

Figure 8: Illustration of the proof of Theorem 5.5 (cases ii.c and ii.d.1)

$\sigma_{l}$ is necessarily timely correct: either $\sigma_{l}$ aborts $\left(k_{e_{1}}>k_{l}\right)$ or $\sigma_{l}$ commits $\left(k_{e_{2}} \leq k_{l}\right)$.

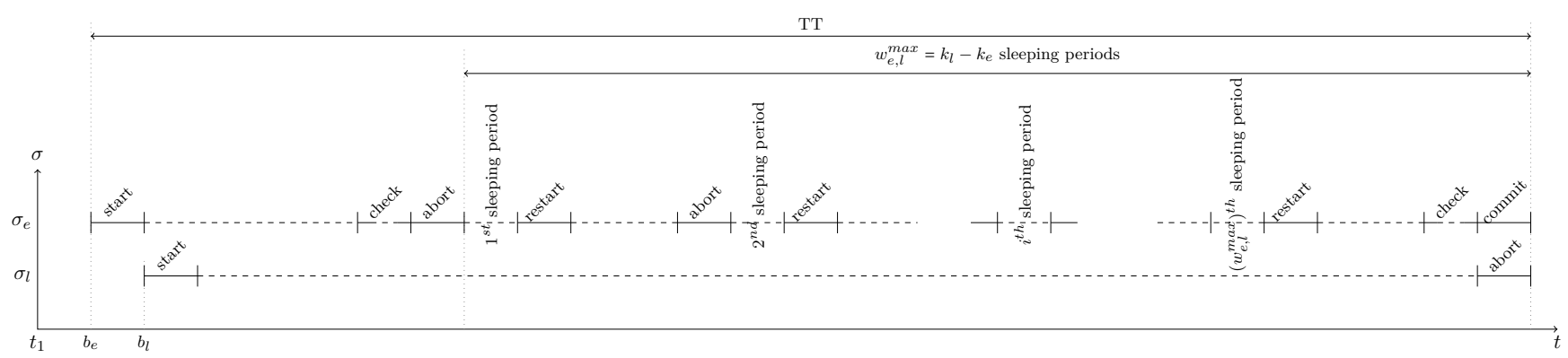

Figure 9: Illustration of the proof of Theorem 5.5 (cases ii.d.3 and ii.d.4)

$\sigma_{l}$ is necessarily timely correct: $\sigma_{e}$ will ultimately abort $\sigma_{l}$ when reaching the maximum number of sleeping periods.

\section{REFERENCES}

[1] A Barros, P Meumeu Yomsi, and L-M Pinho. 2016. Response time analysis of hard real-time tasks sharing software transactional memory data under fully partitioned scheduling. In Proceedings of the11th IEEE Symposium on Industrial Embedded Systems (SIES). IEEE.

[2] A Barros and L-M Pinho. 2014. Non-preemptive scheduling of real-time software transactional memory. In Proceedingsof the 27th International Conference on Architecture of Computing Systems (ARCS). ACM.

[3] S Cotard, A Queudet, J-L Béchennec, Sébastien Faucou, and Yvon Trinquet. 2015. STM-HRT: A Robust and Wait-Free STM for Hard Real-Time Multicore Embedded Systems. ACM Trans. Embedded Comput. Syst. 14, 4 (2015), 1-25.

[4] D Dice, Y Lev, M Moir, D Nussbaum, and M Olszewski. 2009 Early experience with a commercial hardware transactional memory implementation. In Proc. 14th Int. Conf. Archit. Support Program. Lang. Oper. Syst. (ASPLOS). ACM, 157-168.

[5] Son Dinh, Jing Li, Kunal Agrawal, Chris Gill, and Chenyang Lu. 2017. Blocking analysis for spin locks in real-time parallel tasks. IEEE Transactions on Parallel and Distributed Systems 29, 4 (2017), 789-802.

[6] M El-Shambakey and B Ravindran. 2012. STM concurrency control for embedded real-time software with tighter time bounds. In Proceedings of Design Automation Conference (DAC). IEEE.

[7] M El-Shambakey and B Ravindran. 2013. On real-time STM concurrency control for embedded software with improved schedulability. In Proceedings of the 18th Asia and South Pacific Design Automation Conference ( $A S P-D A C)$. IEEE.

[8] Kapali P. Eswaran, Jim N Gray, Raymond A. Lorie, and Irving L. Traiger. 1976. The notions of consistency and predicate locks in a database system. Commun. ACM 19, 11 (1976), 624-633.

[9] Rachid Guerraoui, Maurice Herlihy, Michal Kapalka, and Bastian Pochon. 2005. Robust contention management in software transactional memory. In Proceedings of the OOPSLA 2005 Workshop on Synchronization and Concurrency in Object-Oriented Languages (SCOOL" 05).
[10] Rachid Guerraoui, Maurice Herlihy, and Bastian Pochon. 2005. Toward a theory of transactional contention managers. In Proceedings of the twenty-fourth annual ACM symposium on Principles of distributed computing. ACM, 258-264.

[11] Rachid Guerraoui and Michal Kapalka. 2008. On the correctness of transactional memory. In Proceedings of the 13th ACM SIGPLAN Symposium on Principles and practice of parallel programming. ACM, 175-184.

[12] Theo Haerder and Andreas Reuter. 1983. Principles of transactionoriented database recovery. ACM Computing Surveys (CSUR) 15, 4 (1983), 287-317.

13] Tim Harris, James Larus, and Ravi Rajwar. 2010. Transactiona memory. Synthesis Lectures on Computer Architecture 5, 1 (2010), 1-263.

[14] Maurice Herlihy, Victor Luchangco, and Mark Moir. 2003. Obstruction-free synchronization: Double-ended queues as an example. In Distributed Computing Systems, 2003. Proceedings. 23rd International Conference on. IEEE, 522-529.

[15] Maurice Herlihy, Victor Luchangco, Mark Moir, and William N Scherer III. 2003. Software transactional memory for dynamicsized data structures. In Proceedings of the twenty-second annual symposium on Principles of distributed computing. ACM, 92101.

[16] Ma. Herlihy and J.E.B Moss. 1993. Transactional memory: Architectural support for lock-free data structures. In Proceedings of the 20th Annual International Symposium on Computer Architecture. 289-300.

[17] C Hung, J Yen, L En, S Diestelhorst, M Pohlack, and M Hohmuth. 2010. ASF: AMD64 Extension for Lock-Free Data Structures and Transactional Memory. In Proceedings of the $43 \mathrm{rd}$ Annual IEEE/ACM International Symposium on Microarchitecture. IEEE/ACM, 39-50.

[18] Damien Imbs, José Ramón González De Mendivil Moreno, and Michel Raynal. [n. d.]. On the consistency conditions of transactional memories. Technical Report. Issue 1917, IRISA, University of Rennes, France.

[19] C Jacobi, T Slegel, and D Greiner. 2012. Transactional memory architecture and implementation for IBM system Z. In Proceedings 
of the 45th Annu. IEEE/ACM Int. Symp. Microarchitecture. IEEE/ACM, 25-36.

[20] Eric H Jensen, Gary W Hagensen, and Jeffrey M Broughton. 1987. A new approach to exclusive data access in shared memory multiprocessors. Technical Report. UCRL-97663, Lawrence Livermore National Laboratory.

[21] Xu Jiang, Nan Guan, He Du, Weichen Liu, and Wang Yi. 2020 On the Analysis of Parallel Real-Time Tasks with Spin Locks. IEEE Transactions on Computers (Early Access) (2020).

[22] Lee Kangmin and Jo Heeseung. 2018. ParaTM: Transparent Embedding of Hardware Transactional Memory for Traditional Applications. IEEE Access Journal 6 (2018), 45417-45426.

[23] Toufik Sarni, Audrey Queudet, and Patrick Valduriez. 2009. Realtime support for software transactional memory. In Embedded and Real-Time Computing Systems and Applications, 2009. RTCSA'09. 15th IEEE International Conference on. IEEE, 477485.

[24] William N Scherer III and Michael L Scott. 2005. Advanced contention management for dynamic software transactional memory. In Proceedings of the twenty-fourth annual ACM symposium on Principles of distributed computing. ACM, 240-248.

[25] M Schoeberl, F Brandner, and J Vitek. 2010. RTTM: Realtime transactional memory. In Proceedings of the 2010 ACM symposium on Applied Computing. ACM, 326-333.

[26] Nir Shavit and Dan Touitou. 1997. Software transactional memory. Distributed Computing 10, 2 (1997), 99-116. 